\title{
Opioid Management and Risk Mitigation
}

\author{
Mark Gostine, $M D^{1 *}$, Fred N Davis ${ }^{2}$ and Rebecca Risko ${ }^{2}$ \\ ${ }^{1}$ Clinical Assistant Professor, Michigan State University, College of Human Medicine, USA \\ ${ }^{2}$ ProCare Systems Inc, Grand Rapids, Michigan, USA
}

Over the span of seven months physicians had to reconcile two very different messages regarding pain and the use of opioids issued by institutions that guide healthcare in the United States. First, in June of 2011, The Institute of Medicine (IOM) issued a "Blueprint for Relieving Pain" in America [1]. They rightfully pointed out that chronic pain costs the nation up to $\$ 635$ billion each year in medical treatment and lost productivity. They also stated that "groups of people currently are underdiagnosed and undertreated, and the IOM encourages federal and state agencies and private organizations to accelerate the collection of data on pain incidence, prevalence, and treatments".

Following that perceived call to action for clinicians everywhere, in January of 2012 the Center for Disease Control (CDC), also rightfully, proclaimed "Prescription Drug Overdoses - a U.S. Epidemic" [2]. In the same article, the CDC noted that the increase in unintentional drug overdose death rates in recent years has been driven by increased use of opioid analgesics. Since 2003, more overdose deaths have involved prescription opioid analgesics than heroin and cocaine combined. A significant proportion did not have a prescription in their records for the opioid that killed them; in West Virginia, Utah, and Ohio, $25 \%$ - 66\% of those who died of pharmaceutical overdoses used opioids originally prescribed to someone else. In addition, for every unintentional overdose death related to an opioid analgesic, nine persons are admitted for substance abuse treatment, there are 35 visits to emergency departments, 161 reports of drug abuse or dependence, and 461 reported nonmedical uses of opioid analgesics.

In step with the CDC the FDA has now developed Risk Evaluation and Mitigation Strategies (REMS) for ExtendedRelease and Long-Acting Opioids [3]. Among the tools physicians are now encouraged to use when placing patients on these classes of opioids are:

- The Opioid Risk Tool (ORT) to evaluate patients prior to initiation of opioids

- Numeric Rating Scales to assess baseline pain, typically 0 to 10

- COMM: Current Opioid Misuse Measure - 17 questions to identify misuse and side effects after initiation

- PEG: Evaluates enjoyment of life and consists of three questions from the Brief Pain Inventory

- PHQ-9: Patient Health Questionnaire, nine questions to assess depression

The ORT was developed by Lynn Webster, MD [4] starting with data collected in Utah in the year 2000. It is designed to predict the risk of opioid misuse, obviously a critical assessment we must perform before starting patients on narcotic analgesics. It consists of five questions assigned a total of 26 points. Those questions are:

- Family History of Substance Abuse (possible 10 points)

- Personal History of Substance Abuse (possible 10 points)

- Age (possible 1 point)

- History of Preadolescent Sexual Abuse (possible 3 points if female)

- Psychological Disease (possible 2 points)

The tool's scoring is heavily dependent on patients reporting either a family or personal history of substance abuse. When Doctor Webster conducted his study on 185 patients the technique was manually intensive with face to face interviews. In 2000 the nation was riding the high tech and dot-com boom and Utah typically has the lowest rate of unemployment in the fifty states [5].

In 2010 when we began our efforts at tracking opioid misuse and outcomes across our practice in west Michigan, the nation had experienced a financial retrenchment unequaled in severity since the Great Depression. In many communities in our state we experienced the country's highest unemployment resulting in a marked increase in depression and stress. Opioids became "the new currency" for some of those out of work. It should come as no surprise that the ORT, conceived and developed in Utah in the year

*Corresponding author: Mark Gostine, MD, President Michigan Pain Consultants, Clinical Assistant Professor, Michigan State University, College of Human Medicine, USA, Tel: 616-443-6263

Accepted: February 11, 2019

Published online: February 13, 2019

Citation: Gostine M, Davis FN, Risko R (2019) Opioid Management and Risk Mitigation. J Clin Anesth Pain Manag 3(1):45-50 
2000, may no longer be relevant in Michigan in the year 2010 given the vastly different societal pressures the two states experienced.

Another key difference between our tool and the ORT is that we collected self-reported data on 14,000 patients using iPads versus the face to face interviews employed by Dr. Webster. By 2010 we had developed our own tool called the PRISM ${ }^{\mathrm{TM}}$ Pain Health Assessment (PHA) because there were no digital capture techniques that met our needs. Selfreporting patient surveys, like the Pain Health Assessment (PHA) that we employ, have vastly increased the amount of data captured. However, it would seem easier to misrepresent the truth on a self-reported survey than in a face to face interview where there may be some give and take, along with follow up questions between patient and clinician. A patient highly motivated to divert medications for economic reasons, or seeking to treat depression with opioids, may simply misrepresent the truth with a touch of the iPad. Given these different sets of circumstances, we felt compelled to test the validity of the ORT in our environment.

In addition we conducted a second study to examine outcomes for patients on opioids. We wanted to see how much pain relief patients reported on different doses of narcotic pain relievers. Conducting double blind studies to assess their therapeutic response would be very costly. However, throughout our practice we already have in excess of 10,000 patients who are on various dose levels of narcotics. Since we had been tracking their outcomes since 2010 it was a relatively straightforward proposition to analyze their outcomes and to compare them to the dose level of opioids they were prescribed.

\section{Study Methods}

\section{Clinical setting and patient selection}

Our studies were conducted at Michigan Pain Consultants (MPC), an interdisciplinary community-based pain medicine practice, in Grand Rapids, Ml established in 1984. The practice has seven clinical locations covering a service area of six counties in West Michigan and includes fourteen physicians and six midlevel providers. The data was collected using the $\mathrm{PRISM}^{\mathrm{TM}}$ patient management system, developed by ProCare Systems [6]. The PRISM ${ }^{\mathrm{TM}}$ system is a digital toolbox, containing among other components, the Pain Health Assessment (PHA), a patient reported health outcomes tool. The information is routinely gathered from chronic pain patients in the practice using IRB approved language in the consent forms.

A detailed analysis of observed behaviors associated with narcotic misuse was constructed and correlated with elements in the PHA and ORT. An independent-samples t-test was run on 13,986 de-identified unique patients. Data was further evaluated using Shapiro-Wilks test and Chi-squared tests.

\section{Pain health assessment}

The PHA is a multidimensional structured self-report questionnaire completed on an iPad prior to seeing the physician. It contains core outcomes domains that evaluate the efficacy and effectiveness of treatments, consistent with the recommendations of the Initiative on Methods, Measurement, and Pain Assessment in Clinical Trials (IMMPACT) [7]. The PHA evaluates disease presence, pain characteristics, physical function (e.g. self-care, mobility) and psychosocial function (e.g. emotional and social health dimensions of depression, anxiety, life control and social support) as well as patient satisfaction. It was inspired by the SF-36, TOPS and MPI and includes the ORT. Responses were coded on an 11 point Likert-type scale with 0 "most positive" and 10 "most negative".

\section{ORT correlations with misuse behavior}

Between September 2012 and January of 2013, we identified nearly 400 out of the 13986 unique patients who misused their opioid analgesics [8]. This is only $3 \%$ of our total patient population and is consistent with data from Substance Abuse and Mental Health Services Administration and their results from the 2012 National Survey on Drug Use and Health [9]. Misuse was defined as:

- Testing abnormal on typically more than one urine drug screen (UDS)

- Having an abnormal Michigan Automated Pharmacy Surveillance Report (MAPS)

- Exhibiting behaviors such as calling in early for prescriptions multiple times or being abusive to staff regarding the opioid prescription

Controls were defined as those among the 13,986 who did not misuse their medications. They included all the patients seen in our clinics over this time period. In the chart below (Figure 1) we can see that $66 \%$ of the patients who misused their medications presented with low risk ORT scores. While the ORT was statistically significant in differentiating misusers from patients in the control group, it lacked sensitivity. This observation inspired us to begin our quest for a better tool. Our goal was to examine other domains from the PHA that are less likely to be misrepresented by respondents.

The new opioid risk assessment is called the Narcotic Risk Manager (NRM) [10]. Between September 2012 and July 2013,

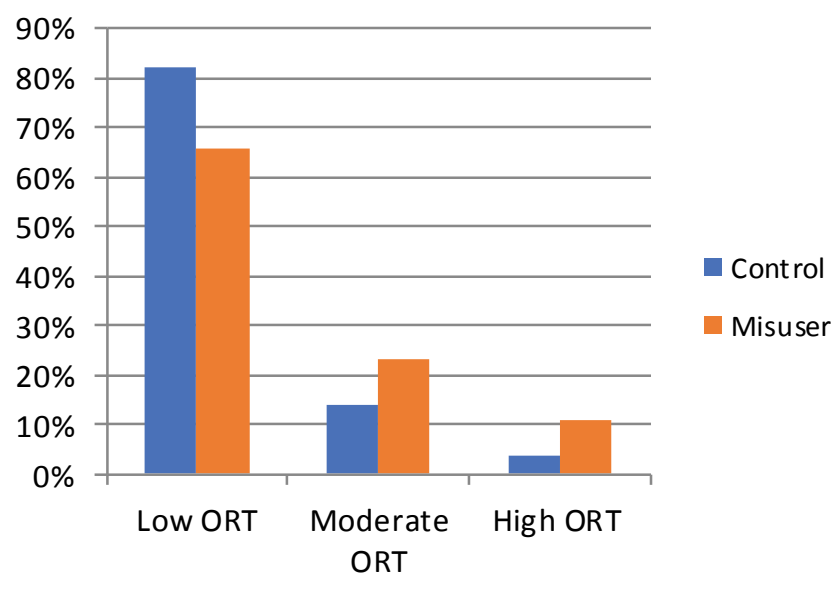

Figure 1: Documented misusers and their risk categories compared to controls assigned by the ORT. 
5,940 patients completing a $\mathrm{PHA}$, and prescribed opiates, were evaluated. A total of $408(6.87 \%)$ were identified as misusers among the opioid prescribed group. The reason the number of patients shrank in the second study from nearly 14,000 to almost 6000 , was because it was conducted on only those patients prescribed opioids. The 14,000 patients in the initial analysis included all patients either on opioids or not. Misuse behavior was defined consistent with the first analysis.

Exploratory analysis identified variables (Table 1) that were significant predictors of opioid misuse, all of which were considered for the final model. Logistic regression was used to model the probability that a patient prescribed opioids would demonstrate misuse behaviors. All two-way interactions were considered for the final model, which was determined using backward selection with $\alpha=0.01$. Seen below are the factors that were initially considered based on our analysis of data in the PHA, a literature review and other risk tools:

Marital Status and history of preadolescent abuse did not stand up to further analysis, so only the significant eight

Table 1: Predictive Factors of Opioid Risk.

\begin{tabular}{|l|l|}
\hline Factor & p-value \\
\hline Gender & 0.005 \\
\hline Smoking Status & 0.0001 \\
\hline Education & 0.0007 \\
\hline History of misuse of drugs or alcohol & 0.0001 \\
\hline Insurance & 0.0042 \\
\hline Number of psychological diseases & 0.0009 \\
\hline Race & 0.0007 \\
\hline Age & 0.0001 \\
\hline History of preadolescent abuse & NS \\
\hline Marital Status & NS \\
\hline
\end{tabular}

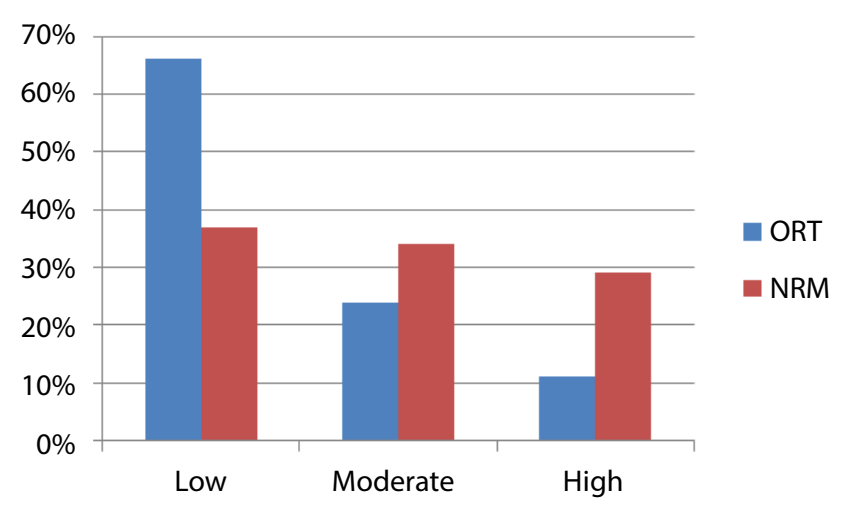

Figure 2: Comparison of documented misusers assigned risk categories in the ORT and NRM. variables make up the final prediction model created using logistic regression. As demonstrated in (Figure 2) two below, the NRM now decreases the number of patients assigned a low risk score that actually misused their prescriptions by almost $50 \%$. While still not perfect it is a substantial improvement, nearly doubling the sensitivity of the ORT.

Looking at the number of patients in this analysis who were on opioids and misused them, the number is very close to $7 \%(408 / 5940)$. We therefore define the average risk of misuse as $7 \%$, moderate risk as twice average $=14 \%$, high risk $=21 \%$, very high as $28 \%$. Clinicians can now readily utilize the NRM online to evaluate their patients with their smart phone, tablet or computer at www.narcoticrisk.com. When using the NRM it takes only seconds to fill in the pertinent information on the eight significant factors above. The patient's risk is reported as both a percent risk and low, moderate or high risk category (Figure 3 ). There are also suggestions on clinical management.

Along with using a risk profile tool like the NRM or ORT, clinicians initiating opioids for potentially long term use, need to chart the patient's pain rating using the classical numeric scale from 0 to 10 . Coincident disease like a bipolar disorder and depression increase the risk of misuse so the PHQ-9 is recommended to evaluate depression. At subsequent visits we want to evaluate the efficacy of opioid therapy, hence the PEG, which is just three questions. Lastly, on an ongoing basis we also have to look at misuse and side effects with the Current Opioid Misuse Measure (COMM), seventeen questions that assess the risk for aberrant medication related behavior among persons prescribed opioids.

How effective are opioids for long term pain control? Our second study we presented at the AAPM conference in February of 2014 looked at pain relief outcomes in patients receiving different doses of narcotics. We know from other studies that high dose narcotic techniques can result in excess hip fractures in the elderly and of course increase risk of misuse and overdose [11]. Pain also adversely impacts a patient's quality of life. We need to balance these two competing imperatives, to do no harm and to relieve pain. Hence we set out to examine the optimal dose of opioids that were associated with the best pain outcomes in our practice.

\section{Population Selection}

Patients were selected based on having had an initial PRISM $^{\text {TM }}$ Pain Health Assessment (PHA) taken between August 1, 2010 and March 31, 2013. In addition, patients who also had a cumulative PHA taken between June 1, 2013 and July 15,2013 were identified. There were a total of 1,032 patients who met criteria (Figure 2); $61.4 \%$ were female, $38.6 \%$ male.

Table 2: Percent Relief and Opioid Dosage Group.

\begin{tabular}{|l|l|l|l|l|}
\hline & Morphine Equivalent Dose & Percent patients on opioid dose & Initial Average Pain & Adjusted mean percent relief \\
\hline No Opioids & & & 4.78 & $64.10 \%$ \\
\hline Low dose & $1-20$ med & $30 \%$ of patients on opioids & 5.32 & $56.80 \%$ \\
\hline Moderate dose & $21-49$ med & $39 \%$ of patients on opioids & 5.53 & $57.60 \%$ \\
\hline High dose & $50-100$ med & $18 \%$ of patients on opioids & 5.86 & $54.01 \%$ \\
\hline Very High dose & $\geq 101$ med & $13 \%$ of patients on opioids & 5.76 & $51.30 \%$ \\
\hline
\end{tabular}


The 532 opioid users were placed into dosage groups (Low, Moderate, High, and Very High) (Table 2) and compared to patients on no opioids. This sample is representative of our practice as a whole.
As we can see from the table above patients on high dose or very high dose opioids had the least amount of adjusted mean pain relief. Potentially this group had more severe pain management issues to begin with. That is why we use

\section{Narcotic Risk Manger (NRM)}

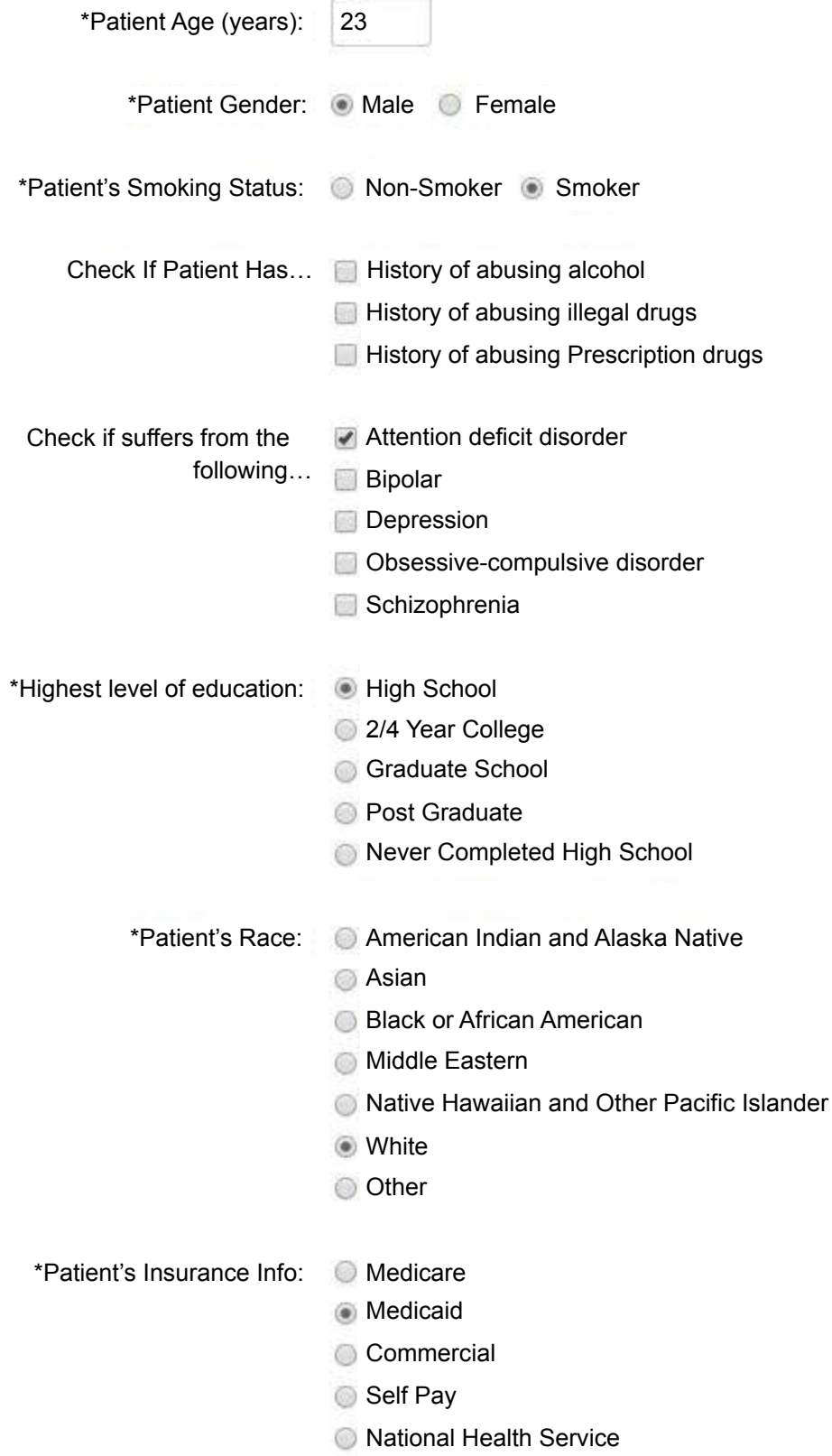

*Indicates Required Field

\section{Very High (34.96\%)}

Figure 3: NRM online to evaluate patient's risk. 
adjusted relief to compare group outcomes. Adjusted relief takes into account the baseline pain level. It is presumed, for example, that obtaining $30 \%$ pain relief for two individuals, one who starts with a pain level of 7 and one who starts with a 5 is comparable.

Why would individuals on higher dose opioids have worse outcomes? High dose opioids have a number of drawbacks:

- Side effects such as constipation, dry mouth, gastric reflux, sedation

- Reduction in sex hormone production fostering menopause in women and impotence in men

- Depression

- Exacerbation of sleep apnea

- Opioid induced hyperalgesia

- Increased risk of hip fractures

- Increased risk of inadvertent overdose.

Certainly when we ask patients to examine their quality of life ( $Q O L$ ) it should not be surprising that the pain control they experience from high or very high dose narcotics may be countered by the side effects. Opioid induced hyperalgesia and tolerance to these medications may explain a great deal of the adverse outcomes. Even though patients become tolerant to the pain relieving qualities of opioids they do not experience a reduction in side effects which remain dose dependent.

The latest explanation for tolerance and hyperalgesia lies with glial cell inflammation. The glial cells provide support to the neurons of the central nervous system. The glial cells surround the neurons that are responsible for nervous system transmission. They hold the neurons in place and supply nutrients and oxygen. It appears that long term administration of opioids cause inflammation of the glial cells and this disrupts the analgesic effects patients derive from narcotics like morphine.

Many side effects however are mediated at the end organ like the gut where morphine causes smooth muscle relaxation leading to constipation and reflux. These side effects are not dependent on glial cell interactions which reside only in the CNS. Hence patients on high dose opioids experience a gradual diminution in the opioids pain relieving qualities but no reduction in side effects.

\section{How to Approach Patients Requiring Opioids}

Using the information above, we can develop a valid approach to patients requiring narcotics. First we must adhere to our primary dictum to "First do no harm". What does this mean in the context of opioid management? We recommend that under most circumstances it is wise to prescribe no more than $100 \mathrm{mg}$ of morphine equivalent dose (MED) per day.

In yet more recent communications from the CDC, they once again pointed out the hazards of opioid prescribing [12]. The overdose risk resulting in death is fourfold greater for patients on more than 100 MED's per day when compared to patients on less than 100 MED's. That is why, with rare exception, it is best to adhere to that dose limit. There may still be circumstances where that is not possible, but patients need to be evaluated closely before starting any narcotic and especially before using a dose larger than 100 MED's.

We should also be aware that as many as $20 \%$ of patients started on opioids in the hospital after trauma remain on opioids for many months. This persistence can lead to lifelong dependency and the potential for misuse. Therefore we need to assess a patient's risk strata at the time of opioid initiation even if we anticipate the use will be for a limited period of time. The NRM can give you a quick risk profile along with recommendations for opioid use. We divide patients into three risk categories low, moderate and high and we suggest guidelines for managing patients in the respective categories. Low Risk

- Pharmacy surveillance report at intake

- No more than 20 mg MED if opioid naïve

- No more than 100 mg MED if opioid tolerant

- Use adjuvants, suggestions:

- Gabapentin, duloxetine for pain

- NSAID's, baclofen, for inflammation and spasm

- Trazadone, Seroquel for sleep and mood disorders

- OK for refills with schedule 3 drugs

- UDS once a year

- No pill counts

- Follow up visits every three months

Moderate Risk

- Pharmacy surveillance report at intake

- No more than 20 mg MED if opioid naïve

- No more than $50 \mathrm{mg}$ if tolerant

- Consider abuse deterrent formulation

- UDS at intake. Twice a year long term

- Pill counts optional

- Use adjuvants, suggestions:

- Gabapentin, duloxetine for pain

- NSAID's, baclofen, for inflammation and spasm

- Trazadone, Seroquel for sleep and mood disorders

- No refills

- Follow up visits every three months

- Consider behavioral assessment

High Risk

- Pharmacy surveillance report at intake

- UDS at intake and up to 4 times per year

- Use pill counts 
- No more than $20 \mathrm{mg}$ MED if opioid naïve, no more than $50 \mathrm{mg}$ if tolerant

- Consider abuse deterrent formulation

- Use adjuvants, suggestions:

- Gabapentin, duloxetine for pain

- NSAID's, baclofen, for inflammation and spasm

- Trazadone, Seroquel for sleep and mood disorders

- No refills

- Follow up visits every three months

- Behavioral assessment mandatory

Is it necessary to evaluate opioid risk in patients with anticipated short term stays and quick recoveries? In this day and age of epidemic prescription drug misuse it is a good idea to have a handle on everyone receiving prescribed narcotics under any circumstances. Our own data shows that $93 \%$ of our patients use their opioids correctly. However, the seven percent who misuse their medications have created a public health emergency that requires those of us in health care to help solve, and one way to start is by employing the proper tools.

\section{References}

1. http://www.nationalacademies.org/hmd/Reports/2011/
Relieving-Pain-in-America-A-Blueprint-for-TransformingPrevention-Care-Education-Research.aspx

2. http://www.cdc.gov/mmwr/preview/mmwrhtml/mm6101a3.htm

3. http://www.fda.gov/drugs/drugsafety/informationbydrugclass/ ucm163647.htm

4. Lynn R Webster, Rebecca M Webster (2005) Predicting aberrant behaviors in opioid-treated patients: Preliminary validation of the opioid risk tool. Pain Medicine 6: 432-442.

5. http://www.bls.gov/web/laus/lauhsthl.htm

6. http://procarepain.com

7. Dworkin RH, Turk DC, Farrar JT, et al. (2005) Core outcome measures for chronic pain clinical trials: IMMPACT recommendations. Pain 113: 9-19.

8. Gostine ML, Davis FN, Gareth Forde, et al. (2013) Correlation of misuse of narcotics with opioid risk tool and pain health assessment: An analysis of 16,000 patients. american academy of pain medicine annual conference.

9. https://nsduhweb.rti.org/respweb/homepage.cfm

10. Gostine ML, Davis FN, Rebecca J Risko, et al. (2014) A new tool for prediction of opioid misuse. American Academy of Pain Medicine Annual Conference.

11. Matthew Miller, Til Stürmer, Deborah Azrael, et al. (2011) Opioid analgesics and the risk of fractures in older adults with arthritis. J Am Geriatr Soc 59: 430-438.

12. https://www.cdc.gov/vitalsigns/opioid-prescribing/index.html

DOI: $10.36959 / 377 / 325$

Copyright: (C) 2019 Gostine M, et al. This is an open-access article distributed under the terms of the Creative Commons Attribution License, which permits unrestricted use, distribution, and reproduction in any medium, provided the original author and source are credited. 